\title{
Diabetes mellitus: A review of its associations with different environmental factors
}

\section{Joshi SK ${ }^{1}$, Shrestha $\mathbf{S}^{2}$}

${ }^{1}$ Associate Professor, Department of Community Medicine, Kathmandu Medical College, Kathmandu, ${ }^{2}$ Medical Officer, Kakani Primary Health Center, Nuwakot

\begin{abstract}
Diabetes Mellitus is a global health problem with a worldwide prevalence of $2.8 \%$ in 2000 . Type 1 diabetes mellitus is an auto immune disorder genetically mediated, while type 2 is more of a life style induced disorder although the role of genetic susceptibility, infections are also equally strong. Many studies have backed up these statements. However, there have been very few researches that show association of diabetes with environmental factors like pollution, exposure to chemicals e.g. mercury, arsenic, psychological condition e.g. depression, stress, and socio-economic conditions e.g. occupation, earnings etc. Recently, the role of these factors in causation and progression of diabetes have received much attention. Thus, this review has been designed to explore more on association of diabetes with physical, socio-economic and psychological environment.
\end{abstract}

Key words: arsenic, diabetes, environment, mercury, occupation, POPs, sleep, socio-economic status, stress

$\mathrm{W}$ orld Health Organization defines Diabetes mellitus as "a chronic disease caused by inherited and/or acquired deficiency in production of insulin by the pancreas, or by the ineffectiveness of the insulin produced. Such a deficiency results in increased concentrations of glucose in the blood, which in turn damage many of the body's systems, in particular the blood vessels and nerves" . The prevalence of diabetes for all age groups worldwide was estimated to be $2.8 \%$ in 2000 and the number is expected to rise to $4.4 \%$ in 2030. Likewise, the figure in number would be 171 million in 2000 estimated to be 366 million in $2030^{2}$. As for Nepal, the estimated number of diabetics in 2000 was 436,000 and it is expected to rise to $1,328,000$ by $2030^{3}$. Diabetes causes about $5 \%$ of all deaths globally each year. $80 \%$ of diabetes deaths are now occurring in low- and middle-income countries ${ }^{4}$.

Type 1 and type 2 are the well known types of Diabetes. Genetic susceptibility, auto-immunity, some viruses and intra-uterine environment have been attributed to diabetes type $1^{5-8}$. Whereas, type 2 is often considered life-style mediated and associated with obesity, high serum level of low density lipoprotein, though role of genetic susceptibility and intra uterine development can also not be denied ${ }^{9-13}$.

But, besides the above mentioned factors, could there be other elements as well, which could cause or manifest diabetes? In fact, it's a very interesting proposition to think of occupation, physical condition of living, stress, or even environmental pollution and their association with diabetes.

\section{Diabetes and its associations}

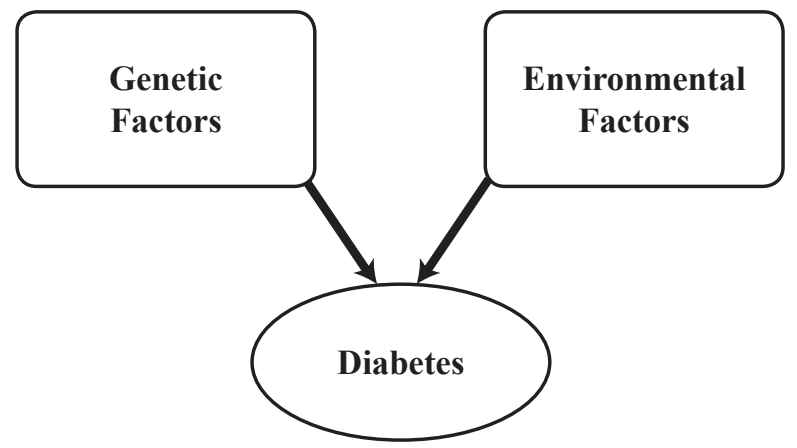

Fig 1: Diabetes and its causations

\section{Diabetes and environmental pollution}

The contribution of persistent organic pollutants (POPs) to causation of diabetes has received little attention until recently ${ }^{14}$. POPs are a group of man-made chemicals. They came into prominence as effective

Correspondence

Dr. Sunil Kumar Joshi

Associate Professor

Department of Community Medicine

Kathmandu Medical College

E-mail: drsunilkj@gmail.com 
pesticides with the introduction of DDT in the 1940s. POPs, including polychlorinated dibenzo-p-dioxins (PCDDs), polychlorinated dibenzofurans (PCDFs), polychlorinated biphenyls (PCBs), hexachorobenzene (HCB) have now been highlighted internationally as chemicals of concern as they were responsible for rapid decline in the number of wild birds and animals ${ }^{15}$. As these chemicals degrade very slowly, they still persist in environment and our food chain, though they were banned long ago. And, considerable amounts of POPs are supposed to be stored in human adipose tissue as well.

In a national health examination survey 1999-2002, Lee $\mathrm{DH}$ et al found that diabetes was strongly positively associated with the serum concentrations of POPs. The results remained same even after careful adjustments for all the possible confounders like age, sex, race and ethnicity, BMI, and waist circumference ${ }^{14}$.

The study was a cross sectional one and it showed only the correlation between POPs serum level and prevalence of diabetes, which does not necessarily be a causal relationship. But, there are certain biological observations which support the causal relationship. Previous studies have shown a POP mediated decrease in glucose transport in vitro and in vivo ${ }^{16}$. Likewise, some chemicals disrupt the ability of body to metabolize fats. This increases body weight and may ultimately precipitate diabetes ${ }^{17}$. However, there are also beliefs that the high concentration of POP might in fact be due to diabetic status. Diabetics already have disorder of metabolism of fat and the POPs are stored in fat, which in turn would not be excreted effectively. Thus, POPs continue to accumulate, which is a secondary phenomenon and not the primary.

Surprisingly, the study showed no association between obesity and diabetes in individuals with low concentration of POPs. But, the association between obesity and diabetes was very intense among those with high levels of POPs. This finding might imply that all the risk of diabetes due to obesity might actually be due to the POPs which were accumulated in the fats of obese people - obesity merely serving as a vehicle for POPs $^{18}$. This would be a completely new and astounding hypothesis.

To cover up for the inability of the previous study to establish causal relationship, Rignell-Hydbom et al conducted a study among a cohort of women from the southern part of Sweden ${ }^{19}$. A baseline evaluation of all the women in that cohort was made. Later, those who developed diabetes were taken as cases and suitable age, BMI matched women were selected as controls. There were altogether 371 cases. Two biomarkers for
POP exposure, 2,29,4,49,5,59-hexachlorobiphenyl (CB-153) and 1,1-dichloro-2,2-bis (p-chlorophenyl)ethylene ( $p, p 9-D D E)$, in serum were collected before the cases had type 2 diabetes diagnosed. They found that high serum concentrations of p,p 9-DDE is a strong risk factor for developing type 2 diabetes later in life. A fivefold statistically significant increased risk was observed among the individuals with the longest follow-up.

Likewise, a study in Taiwan also confirmed this association. A mass poisoning occurred in central Taiwan after a quantity of rice-bran oil ingested in 1978-1979 was later found to be contaminated with PCBs and their heat-degraded byproducts ${ }^{20}$. By the end of February 1983, there were 2,061 recorded cases of PCB poisoning, based on the symptoms and pathology of the illness, such as abnormally high levels of blood PCBs. This particular group of people formed a study cohort, called Yucheng. After 24 years, this study was conducted to examine the hypothesis that raised levels of PCBs and dibenzofurans (DFs) might be associated with an increased risk of diabetes.

Individuals for the background-exposed groups were recruited in 1992 and matched against the Yucheng subjects for neighborhood (the same back in 1979), sex, age (no more than 3 years' difference), and exposure to POPs, such that none of the control individuals were in the original registration cohort. Between 1993 and 2003, a morbidity follow-up of the exposed subjects and their reference group was done with trained interviewers blinded to exposure status. After all exclusions, 378 Yucheng and 370 matched reference subjects remained for analysis within the study.

In this study, mean PCBs in the Yucheng subjects were about 40- to 50-fold those of the reference group. The AOR (OR adjusted for age and BMI in women and age, BMI, cigarette smoking, and alcohol drinking in men) of diabetes for the Yucheng cohort relative to the reference group remained significant in women (OR $2.1 ; \mathrm{P}<0.05)$. They also found an age-adjusted $\mathrm{OR}$ of $6.4(\mathrm{P}<0.05)$ in women aged more than 65 years who received diabetes therapy, which rose to $6.6(\mathrm{P}<0.05)$ after further adjustment for BMI.

Thus they found that diabetes was twice as prevalent in Yucheng women who had been exposed to PCBs and PCDFs during the 1978-1979 poisoning as it was in the reference population in the long-term cohort study. The AOR significantly increased to 2.5 for those requiring therapy for diabetes and to 5.5 for those with chloracne, a condition symptomatic of POP poisoning. Similar results were found in a recent study among a large cohort of Great Lakes Sport fish consumers, 1992$2005^{21}$. In this cohort study, the POP body burdens in 
1994-1995 were compared with incident diabetes from 1995 to 2005. All in all, they noted consistent, doserelated associations of DDE with incident diabetes.

Other studies have also shown the association of POPs with diabetes. Rylander and colleagues had analyzed the concentrations of hexachlorobiphenyl and dichloro-2,2bis (p-chlorophenyl)-ethylene (p,p-DDE, a breakdown product of DDT) in serum of 196 men and 184 women in a fishing community in Sweden ${ }^{22}$. Both compounds were strongly associated with diabetes. Likewise, studies in Belgium ${ }^{23}$, the US ${ }^{24}$ have also shown relation between chemical exposure and symptoms of diabetes.

Since, POPs are widespread in our environment; their effect on diabetes status has to be seriously studied as the effect of any other factor like obesity, and dietary pattern.

\section{Diabetes and arsenic exposure}

Not only POPs, but arsenic has also been linked with diabetes. Many studies have proved dose-response relationship between arsenic in drinking water and prevalence and mortality of diabetes ${ }^{25,26}$.

Arsenic has been speculated to increase oxidative stress thus inducing both insulin-dependent and non-insulindependent diabetes ${ }^{27}$. It also reduces the anti-oxidant capacity, depletes glutathione and thus induces insulin resistance ${ }^{28}$.

Lai et al studied 891 adults residing in southern Taiwan where arseniasis was epidemic in $1988^{25}$. They found the prevalence of diabetes mellitus among the residents in that area was twice that among residents in Taipei city and the Taiwan area. There was also a dose-response relation between cumulative arsenic exposure and prevalence of diabetes mellitus, with an odds ratio of 6.61 and 10.05 respectively for those who had a cumulative arsenic exposure of 0.1-15.0 and greater than $15 \mathrm{ppm} / \mathrm{year}$ compared to those who were unexposed.

Furthermore, Chiu et al looked into effect of arsenic on the mortality due to diabetic complications ${ }^{29}$. In Taiwan, in the study area, before 1960, majority of people drank artesian water which was high in arsenic concentration. During the same period, a peripheral vessel disease termed black foot disease was endemic in that area and the prevalence of DM was very high. After tap water system was established in early 1960s, people stopped using artesian wells for drinking water. Thus, the researchers then looked into the trend of mortality among the residents there over nearly three decades to see if there was any change in the mortality pattern, now that the arsenic rick water was replaced.
They found that the mortality due to diabetic complications decreased with the improvement in water supply system. This strengthened the belief that prolong exposure to arsenic could induce diabetes. But, the decline in mortality was found only in women and not in men - the researchers believe it might have been because of the fact that women are more susceptible to arsenic and men in that area might have had more risk factors than the women in the same area. Whatever the arguments are, the association of arsenic with diabetes was once again proven.

High prevalence of diabetes has also been reported among copper smelter workers ${ }^{30}$ and art glass industry workers $^{31}$. The common risk factor was higher degree of exposure to arsenic.

\section{Diabetes and mercury exposure}

Mercury is a well-known toxic agent that induces oxidative stress and produces various types of cell and tissue damage. Researchers have also found its association with diabetes as well.

Shing-Hwa Liu and colleagues claimed that the mercury compound present as a contaminant in some seafood can damage insulin-producing cells in the pancreas ${ }^{32}$. In their experiments, they exposed cell cultures of insulinproducing beta cells to methylmercury (MeHg) at about the same levels as people would consume in fish. The number of viable cells was reduced 24 hour after $\mathrm{MeHg}$ treatment in a dose-dependent manner with a range from 1 to 20 microM. MeHg could also suppress insulin secretion in HIT-T15 cells and isolated mouse pancreatic islets. After 24 hour of exposure to $\mathrm{MeHg}$, HIT-T15 cells had a significant increase in mercury levels with a dose-dependent manner. Moreover, $\mathrm{MeHg}$ displayed several features of cell apoptosis including an increase of the sub-G1 population and annexin- $\mathrm{V}$ binding. Thus, methylmercury-induced oxidative stress causes pancreatic beta cell apoptosis and dysfunction.

However, Shing-Hwa's findings come opposite to a finding from previous epidemiological study which stated that residential history in a methyl mercury polluted area does not increase the risk for developing diabetes $^{33}$. The study was a population based cross sectional mass screening survey. A case-control study was designed to estimate the role of various risk factors including methyl mercury exposure for diabetes mellitus. A total of 1,087 persons older than 40 years were examined. The prevalence rate of the diabetes mellitus was $8.4 \%$ in males and $5.3 \%$ in females. The odds ratio of residential history in a methyl mercury high polluted area was 0.58 . The study concluded that the prevalence of the diabetes mellitus in methyl mercury polluted area was not increased. 
Diabetes, occupation and socio-economic status There is little information about the prevalence of diabetes among workers in different occupations or people from different socio-economic backgrounds.

S'anchez-chaparro et al did a study among 259,014 Spanish workers to look for the prevalence of metabolic syndrome and its components ${ }^{34}$. Workers were divided into manual workers and non manual workers. Nonmanual works included managerial, clerical and intellectual works whereas manual works included salesperson, agriculture and fishing workers, security service workers, craftsmen and women, machine installers and operators.

They found that among female subjects, prevalence of metabolic syndrome was higher in manual workers than in non-manual workers, lowest prevalence among females being among general managers and government administrators. In contrast among male subjects, prevalence of metabolic syndrome was similar in manual and non-manual workers. The highest prevalence was however, found among machinery operators, installers and assemblers.

Likewise, there was similar study which looked into the prevalence of metabolic syndrome among 7256 active workers in a large car factory and a department store ${ }^{35}$. The overall prevalence of metabolic syndrome was $10.2 \%$. It was significantly higher in men than women, $8.7 \%$ vs $3.0 \%$ respectively at $95 \%$ confidence level. Prevalence was highest in manual workers $(11.8 \%)$, and lower in office workers $(9.3 \%)$ and managers $(7.7 \%)$. This indicates difference in prevalence of metabolic syndrome with difference in social classes.

This finding has been supported by another study as well, which associated household income and occupation with metabolic syndrome ${ }^{36}$. It found that there is an inverse relationship between household income and risk of metabolic syndrome among the women. Of course, low income might mean poor and unhealthy dietary habits, poor life style and health check-ups. But, strikingly, this inverse relationship remained statistically significant even after adjustment on lifestyle factors. This suggests that low income means an unfavorable social and economic environment, which increases the risk of metabolic syndrome. This was not found among men, however. The other finding of the study was high incidence of metabolic syndrome among both men and women with low educational level and duration of schooling and among those who lived in rented apartments. The hazardous effect of socio-economic status among women was also evidenced by another study $^{37}$.
Thus, low socio-economic status is a strong predictor of diabetes, one more study providing evidence for $\mathrm{it}^{38}$. It studied 975 white and 418 african-american adults aged 35 to 54 years. African-American participants had lower socioeconomic status and were more obese with greater central adiposity. Compared to whites, they were twice more likely to get diabetes $(\mathrm{OR}=2.38)$. Likewise, the prevalence of diabetes in African-Americans was greatest in individuals with low socio-economic status $(\mathrm{OR}=4.09)$.

\section{Diabetes and environment}

The effect of environment on inducing diabetes is not a new discussion now ${ }^{39}$. A clear evidence has been found among migrants who moved from a low diabetes prevalence area to a high prevalence area.

There has been a stepwise increase in the prevalence of obesity and thus diabetes in Blacks along the path of migration i.e. $5 \%$ in Nigeria, $23 \%$ in Jamaica and $39 \%$ in the USA. The same trend is also noted among south Asian migrants who developed nearly four times increase in prevalence rate of diabetes type 2 compared to the back-home inhabitants.

These increases in prevalence were found to coincide with some factors which are determinants of diabetes. They are nutrition transition, physical inactivity, geneenvironment interaction, stress and other factors such as ethnic susceptibility. Thus change in life style is a strong determinant of diabetes mellitus type 2 .

Effect of environment has been shown true even for type 1 diabetes, which is mostly supposed to be autoimmune and genetic disease. A study was conducted among children who migrated from Pakistan to Bradford, UK. The incidence of type 1 diabetes mellitus was noted among them. The incidence increased from 3.1/100000 per year in $1978-81$ to $11.7 / 100000$ per year in $1988-90$, where as the incidence for the native children remained constant at $10.5 / 100000$ per year ${ }^{40}$. Even striking was the fact that, the increase was noted despite the migrants sticking to their own dietary habits of Pakistan. There was no change in diet at all. The researchers thus concluded the change that brought about such rise in incidence could be exposure to viruses, which are uncommon in Pakistan. Also, climate might have its role as diabetes seems rare in hot countries and is increasingly common in cooler northern European countries.

\section{Diabetes and ethnicity}

There is little doubt that change in lifestyle - more urban style - induces higher incidence of diabetes. But, studies have shown that the increase in incidence of diabetes for the same change in lifestyle differs among different 
ethnic groups of people ${ }^{41}$. Asian Indians had a higher prevalence of diabetes than the European descendants, though they have similar life style and environmental exposure. Migrant Indians were shown to have increased insulin resistance and hyperinsulinemia compared to Europeans.

Another study has also shown higher risk of diabetes among Asian population who get obese compared to other ethnic groups who were equally obese ${ }^{42}$. In this study, researchers measured insulin levels and compared the amount of total body fat to lean mass in 828 men and women of Aboriginal, Chinese, European and South Asian origin. They found increased insulin resistance and diabetes among the south Asians. In addition, they also found that south Asians had both higher fat mass and lower muscle mass.

They concluded that it's the distribution of fat and muscle mass that determines ones susceptibility to diabetes and that the distribution of fat and muscle is in turn determined by the ethnic background.

\section{Diabetes and sleep}

Studies have shown relationship between sleep and diabetes - both long and short sleepers being at greater risk for diabetes. Results showed that the adjusted odds ratio was 1.24 for diabetes associated with short sleep (five hours per night or less) and 1.48 for diabetes associated with long sleep (nine or more hours per night). Specifically, individuals sleeping for more than eight hours per night may be particularly vulnerable. The conclusions were based on a study that involved data from 29,818 individuals who completed the 2005 National Health Interview Survey.

Another study has also proved the association ${ }^{43}$. Researchers in this study subjected a group of healthy middle-aged men and women to two controlled 14-day periods of sedentary living with free access to food and 5.5 or 8.5 hour bedtimes. When the subjects had their bedtimes decreased from 8.5 hours to 5.5 hours they showed changes in their response to two common sugar tests, which were similar to those seen in people with an increased risk of developing diabetes. This association is very significant because, decreased sleep has become a part of urban life which is already made a danger for diabetes because of its dietary practice, low physical activity life and obesity.

\section{Diabetes and stress}

Stress has been reported to increase blood glucose and decrease insulin activity ${ }^{44}$. Golmohammadi et al carried out a study to look into association of stress with diabetes mellitus type $2^{45}$. They used a 55 item questionnaire inquiring about work condition, job environment, and personal feelings that workers have at work. They studied 123 diabetic employees and 150 non-diabetic control subjects and found a significant difference in stress among the two groups.

Of course, the association does not necessarily mean causation and either of the two factors - stress and diabetes - could be a cause or an effect. Stress could be a result of diabetic status or it could also be a cause for diabetes, which the study concluded.

Agardh et al showed association of stress with type 2 diabetes mellitus among middle aged Swedish women ${ }^{46}$. Likewise Mooy et al in Netherlands have also shown that stressful life events like death of partner, moving from a house are associated with type 2 diabetes mellitus ${ }^{47}$. Furthermore, Cobbs et al have also shown increased risk of diabetes mellitus among air traffic controllers, their job requiring high levels of concentration and attention $^{48}$.

As mentioned earlier, stress could well be a result of diabetes. Empirical studies suggest that depression is more prevalent among adults with diabetes than among the general population. To date, the reasons for the higher prevalence rates of depression in diabetic patients are not yet fully understood. There are two dominant hypotheses: 1) it results from biochemical changes directly due to the illness or its treatment and 2) it results from the psychosocial demands or psychological factors related to the illness or its treatment. The former hypothesis has also been referred to as a mood disorder due to a medical condition for which specific criteria have been formulated in Diagnostic and Statistical Manual of Mental Disorders. Both hypotheses are not mutually exclusive ${ }^{49}$.

\section{Conclusions}

There is no doubt on the association of diabetes mellitus type 2 with unhealthy lifestyle practices like physical inactivity, obesity, low fiber diet etc. Only these factors are stressed much most of the time, while evaluating the diabetic status and its management. On the other hand, several other factors are underestimated and overlooked, which in fact do carry very significant role in causation, manifestation and progression of diabetes. These factors include stress, environmental pollution, chemical exposure, occupation, ethnicity and low socioeconomic status. Thus, these factors also need equal emphasis if we are to control and effectively manage diabetes. 
References

1. WHO. Diabetes mellitus. [Cited 2010 Jan 10]. Available from: http://www.who.int/ diabetesactiononline/diabetes/en/

2. Wild S, Roglic G, Green A, Sicree R, King H. Global prevalence of Diabetes. Estimates for the year 2000 and projections for 2030. Diabetes care 2004;27:1047-53.

3. WHO. 10 facts on Diabetes. [Cited 2010 Jan 10]. Available from: http://www.who.int/ diabetes/facts/world figures/en/index5.html

4. WHO. 10 facts on diabetes. [Cited 2010 Jan 10]. Available from: http://www.who.int/features/ factfiles/diabetes/08_en.html

5. Atkinson MA, Maclaren NK. The pathogenesis of insulin-dependent diabetes mellitus. N Engl J Med 1994;331(21):1428-36.

6. Dahlquist GG, Patterson C, Soltesz G. Perinatal risk factors for childhood type 1 diabetes in Europe. The EURODIAB Substudy 2 Study Group. Diabetes Care 1999;22(10):1698-702.

7. Frier BM, Fisher BM. Diabetes Mellitus. In: Davidson's principles and practice of medicine. Haslett CE, Chilvers ER, Boon NA, Colledge NR, Hunter JAA, editors. Edinburgh: Churchill Livingstone; 2005. p. 653.

8. Nakayama M, et al. Prime role for an insulin epitope in the development of type 1 diabetes in NOD mice. Nature 2005;435(7039):220-3.

9. DeFronzo RA and Ferrannini E. Insulin resistance. A multifaceted syndrome responsible for NIDDM, obesity, hypertension, dyslipidemia, and atherosclerotic cardiovascular disease. Diabetes Care 1991;14(3):173-94.

10. Dyck RF, Klomp H, Tan L. From "thrifty genotype" to "hefty fetal phenotype": the relationship between high birthweight and diabetes in Saskatchewan Registered Indians. Can J Public Health 2001;92(5):340-4.

11. Hofman PL, etal.Premature birth and laterinsulin resistance. N Engl J Med 2004;351(21):217986.

12. Rich-Edwards JW, et al. Birthweight and the risk for type 2 diabetes mellitus in adult women. Ann Intern Med 1999;130(4 Pt 1):278-84.

13. Sullivan PW, et al. Obesity, inactivity, and the prevalence of diabetes and diabetes-related cardiovascular comorbidities in the U.S., 20002002. Diabetes Care 2005;28(7):1599-603.

14. Lee DH, et al. A strong dose-response relation between serum concentrations of persistent organic pollutants and diabetes: results from the National Health and Examination Survey 19992002. Diabetes Care 2006;29(7):1638-44.
15. Abelsohn A, Sanborn MD, Weir E, et al. Identifying and managing adverse environmental health effects. 5. Persistent organic pollutants. CMAJ 2002;166:1549-54.

16. Olsen H, Enan E, Matsumura F. Regulation of glucose transport in the NIH 3T3 L1 preadipocyte cell line by TCDD. Environ Health Perspect 1994;102:454-8.

17. Jones OAH, Maguire ML, Griffin JL. Environmental pollution and diabetes: a neglected association. Lancet 2008;371:287-8.

18. Porta M. Persistent organic pollutants and the burden of diabetes. Lancet 2006;368:558-9.

19. Rignell-Hydbom A, Rylander L, Hagmar L. Exposure to persistent organochlorine pollutants and type 2 diabetes mellitus. Hum Exp Toxicol 2007;26(5):447-52.

20. Wang SL, et al. Increased risk of diabetes and polychlorinated biphenyls and dioxins: a 24year follow-up study of the Yucheng cohort. Diabetes Care 2008;31(8):1574-9.

21. Turyk M, et al. Organochlorine exposure and incidence of diabetes in a cohort of Great Lakes sport fish consumers. Environ Health Perspect 2009;117(7):1076-82.

22. Rylander L, Rignell-Hydbom A, Hagmar L. A cross-sectional study of the association between persistent organochlorine pollutants and diabetes. Environ Health 2005;4:28.

23. Fierens S, Mairesse $\mathrm{H}$, Heilier JF, et al. Dioxin/polychlorinated biphenyl body burden, diabetes and endometriosis: findings in a population-based study in Belgium. Biomarkers 2003;8:529-34.

24. Fujiyoshi PT, Matsumura F, et al. Molecular epidemiologic evidence for diabetogenic effects of dioxin exposure in US air force veterans of the Vietnam war. Environ Health Perspect. 2006;114:1677-83.

25. Lai MS, Hsueh YM, Chen CJ, et al. Ingested inorganic arsenic and prevalence of diabetes mellitus. Am J Epidemiol 1994;139:484-92.

26. Wang SL, Chiou JM, Chen CJ, et al. Prevalence of Non- Insulin-Dependent diabetes mellitus and related vascular diseases in southwestern arseniasisendemic and nonendemic areas in Taiwan. Environ Health Perspect 2003;111:1559.

27. Longnecker MP, Daniels JL. Environmental contaminants as etiologic factors for diabetes. Environ Health Perspect 2001;109(suppl 6):871-6. 
28. Suzuki KT, Tomita T, Ogra Y, Ohmichi M. Glutathione-conjugated arsenicsin the potential hepato-enteric circulation in rats. Chem Res Toxicol 2001;14:1604-11.

29. Hui-Fen Chiu, Chang CC, Tsai SS, Yang CY. Does Arsenic Exposure Increase the Risk for Diabetes Mellitus? J Occup Environ Med 2006;48(1):63-7.

30. Rahman M, Axelson O. Diabetes mellitus and arsenic exposure: a second look at case-control data from a Swedish copper smelter. Occup Environ Med 1995;52:773-4.

31. Rahman M, Wingren G, Axelson O. Diabetes mellitus among Swedish art glassworkersan effect of arsenic exposure? Scand J Work Environ Health 1996;22:146-9.

32. Chen YW, Huang CF, Tsai KS, et al. Methylmercury induces pancreatic beta-cell apoptosis and dysfunction. Chem Res Toxicol 2006;19(8):1080-5.

33. Futatsuka M, Kitano $T$, Wakamiya J. An epidemiological study on diabetes mellitus in the population living in a methyl mercury polluted area. J Epidemiol 1996;6(4):204-8.

34. Sanchez-Chaparro MA, Calvo-Bonacho E, González-Quintela A, et al. Occupation-related differences in the prevalence of metabolic syndrome. Diabetes Care 2008;31(9):1884-5.

35. Alegria E, et al. Prevalence of metabolic syndrome in the Spanish working population: MESYAS registry. Rev Esp Cardiol 2005;58(7):797-806.

36. Dallongeville $\mathrm{J}$, et al. Household income is associated with the risk of metabolic syndrome in a sex-specific manner. Diabetes Care 2005;28(2):409-15.

37. Robbins JM, et al. Socioeconomic status and diagnosed diabetes incidence. Diabetes Res Clin Pract 2005;68(3):230-6.

38. Brancati FL, et al. Diabetes mellitus, race, and socioeconomic status. A population-based study. Ann Epidemiol 1996;6(1):67-73.
39. Misra A, Ganda OP. Migration and its impact on adiposity and type 2 diabetes. Nutrition 2007;23(9):696-708.

40. Bodansky $\mathrm{HJ}$, et al. Evidence for an environmental effect in the aetiology of insulin dependent diabetes in a transmigratory population. BMJ 1992;304(6833):1020-2.

41. Abate N, Chandalia M. Ethnicity, type 2 diabetes \& migrant Asian Indians. Indian J Med Res 2007;125(3):251-8.

42. Lear SA, et al. Ethnic variation in fat and lean body mass and the association with insulin resistance. J Clin Endocrinol Metab 2009;94(12):4696-702.

43. Nedeltcheva AV, et al. Exposure to recurrent sleep restriction in the setting of high caloric intake and physical inactivity results in increased insulin resistance and reduced glucose tolerance. J Clin Endocrinol Metab 2009;94(9):3242-50.

44. Surwit RS, Feinglos MN, et al. Stress and diabetes mellitus. Diabetes care 1992;15:141322.

45. Golmohammadi R, et al. Relationship between occupational stress and non-insulin-dependent diabetes in different occupation in Hamadan (West of Iran). J Med Sci 2006;6(2):241-4.

46. Agardh EE, Ahlbom A, Tomas E, Suad G, Valdemar H, Anders JN, et al. Work stress and low sense of cohorence is associated with type 2 Diabetes in middle aged swedish women. Diabetes Care 2003;26:719-24.

47. Mooy JM, de Vries H, Grootenhuis PA, Bouter LM, Heine RJ. Major stressful life events in relation to prevalence and undetected type 2 diabetes. Diabetes Care 2002;3:197-201.

48. Cobbs S, Rose RM. Hypertension, peptic ulcer and diabetes in air traffic controllers. JAMA 1973;224:489-92.

49. Talbot F, Nouwen A. A review of the relationship between depression and diabetes in adults: is there a link? Diabetes Care 2000;23(10):155662 . 